series of domains, notably 'Self-Leadership' and 'Collaborating \& Influencing'. Distinct components within the SCLF programme were felt to be especially useful; namely 'Leadership Development Days', Coaching and the shadowing of medical leaders. The evaluation identified a need to provide ongoing support for Fellows returning to clinical training, with many Fellows citing time constraints, a lack of colleague understanding and unsupportive workplace cultures as particular challenges.

Conclusion The evaluation will help inform the future development of the SCLF programme and may offer useful learning regarding the format and content of similar clinical leadership programmes.

\section{WOMEN SPEAKERS IN HEALTHCARE: TAKING STEPS TOWARDS EQUAL GENDER REPRESENTATION AT ALL HEALTH CARE CONFERENCES AND EVENTS}

Rose Penfold, Katie Knight*, Lucia Magee*, Greta McLachlan*, Nada Al-Hadithy. National Medical Director's Clinical Fellow, UK

10.1136/leader-2019-FMLM.12

Women represent an increasing proportion of the health and social care workforce, yet remain underrepresented on boards and in senior NHS leadership positions. ${ }^{1}$ There is thus a high probability that conference organisers trying to convene wellknown speakers will find themselves with a 'manel' (an allmale panel of speakers), or a significantly gender-imbalanced lineup. Conferences offer unrivalled opportunities to showcase diversity and inclusivity, to network and as a lever for cultural and organisational change. Speakers and panels should model what we want our future health and social care workforce to look like.

Token women representatives or waiting for change are insufficient. Women Speakers in Healthcare was co-founded by 5 NHS healthcare professionals, with a vision to ensure balanced gender representation at all healthcare conferences and events, with parity of opportunity for all. We are actively promoting and raising the profile of women speakers by:

1. Creating the UK's largest database of women speakers in healthcare;

2. Providing development and training opportunities to inspire and enable women and

3. Engaging women speakers and male allies through networking and collaboration.

To-date, WSH has 1761 Twitter followers, 390 women speakers signed up to the database and has facilitated conference-speaker connections. We encourage all forms of diversity, are actively engaging male allies and encourage speakers and supporters to nominate other women speakers. We aspire to continue to grow the database, facilitate further speaker-organiser connections and ultimately to ensure that we never again hear the words 'we regret that the conference organisers couldn't find a suitable female speaker for the panel...'.

Website: www.womenspeakersinhealthcare.co.uk

Twitter: @womenspeakersHC

\section{REFERENCES}

1. NHS Women on Boards by 2020. Exeter Business School, NHS Improvement and NHS Employers. Published 29 Mar 2017. Available at: https://www.nhsemployers. org/-/media/Employers/Publications/NHS-Women-on-Boards-report.pdf. Accessed 30 Mar 2019.

\section{Enhancing Your Leadership and Management Skills}

\section{NURTURING MENTORSHIP IN HEALTH CARE ORGANIZATIONS: 'HOW DO YOU KNOW'}

${ }^{1}$ Francesco Locati* ${ }^{1}$ Roberto Cosentina, ${ }^{1}$ Patrizia Bertolaia, ${ }^{1}$ Gianluca Vecchi, ${ }^{2}$ Giulia Martinelli, ${ }^{3}$ Marco Salmoiraghi. ' General Directorate Health Care Trust Unit Seriate, Italy; ${ }^{2}$ Regione Lombardia, Milan, Italy; ${ }^{3}$ General Directorate Welfare, Regione Lombardia, Milan, Italy

\subsection{6/leader-2019-FMLM.13}

Health Care Organizations are properly considered the most complex ones and there is a growing attention at a complexity perspective in health policy. Leading complex organizations is a crucial task, moving away from a trait and personality background towards the relevance of leadership styles and behaviours.

Based on this assumption, we planned the development of an effective model for leading groups or organizations in health care in order to achieve better outcomes, that arises from a structured observation of Health Care CEOs working in their context. The main clue was the importance of specific behaviour patterns that can be elicited through modeling processes.

Modeling process enables people in the groups and learning organizations to cope through scheduled intervision to verify improvements and to produce continuous feedback. Different techniques based on the so-called 'sleight of mouth' procedure were selected to elicit specific leadership patterns, through an observational study, and comparing main indicators of performance, included in Regione Lombardia and Sant'Anna of Pisa networks.

Among the set of behavior patterns investigated through the lens of 'sleight of might' technique were: not knowing, active listening, relationships, giving answers immediately, seeking feedback, values bridging, easiness, reframing, sense making, utilizing 'strange' attractors, interpersonal management tactics. The conclusive results of the benchmarking analysis and the patterns are ongoing. Impact is valued through the awareness of different levels of learning, recognizing specific patterns of performance, modeling and acting strategies for change.

Learning groups and organizations are powerful setting of improvement, particularly in health care where many variables are at stake. The analysis of the patterns to be elicited related to performance represents a platform for coping and reproducing successful strategies in the realm of leadership.

\section{Leading Innovation and Improvement}

\section{WOMEN EMPOWERING WOMEN}

Sarb Clare, Sarah Faloon, May Yan. SWBH NHS Trust

10.1136/leader-2019-FMLM. 14

$76 \%$ of NHS workers are female yet only $36 \%$ are in senior positions. Female doctors dominate medical school with the majority at $56 \%$, however only $36 \%$ are Medical Consultants, 
$11 \%$ are surgeons and only $25 \%$ are Medical Directors. Women bring unique qualities within leadership such as compassion, innovation, sincerity and empathy, which are key for balance to any team.

To address this issue a Deputy Medical Director, RCP tutor and CMT delivered a one day national free conference aimed at medical female doctors entitled 'Women Empowering Women' to enable, educate, inspire and empower women to apply for senior positions within the NHS. The day took place at the Sandwell and West Birmingham NHS Trust. Delegates from across the UK attended.

Women bring unique qualities within leadership such as compassion, innovation, sincerity and empathy, which are key for balance to any team.

The day involved inspiring talks from females in leadership and management roles, including the President of the Women's Federation as well as workshops including Research and Academia, Management and Leadership as well as Teaching and Education.

Further workshops included having babies and less that full time working as well as self care.

The key aspects that were being addressed was

1. Role modeling - showcasing individuals who are in senior leadership positions and sharing their journey 'If uou can see it you can be it'.

2. Processes - maternity rights to application of jobs were addressed.

3. Mindset- positivity and surrounding yourself with energy and removing the imposter syndrome mentality.

A workshop addressing self - defence and dealing with inappropriate behavior in the workplace was also very popular and stories were heard as well as strategies moving forward to address this.

From this day many initiatives are being generated at our Trust including a Women's network and mentoring scheme for females.

\section{Enhancing Your Leadership and Management Skills}

\section{THE FOUNDATION FORUM: A PLATFORM FOR DEVELOPING LEADERSHIP IN MEDICAL EDUCATION AMONGST FOUNDATION TRAINEES}

${ }^{1}$ Murtaza Kadhum*, ${ }^{1}$ Pierre Sinclair, ${ }^{2}$ Chetan Narshi. ${ }^{1}$ Oxford University Clinical Academic Graduate School, Oxford University; ${ }^{2}$ Department of Rheumatology, Stoke Mandeville Hospital, Buckinghamshire NHS Foundation Trust

10.1136/leader-2019-FMLM.15

Foundation forums have been adopted over the last few years to provide representation for newly qualified doctors. These forums, however, do not develop essential leadership, management and quality improvement skills for those recruited, key attributes that have been formally identified by recent GMC guidance. A foundation forum was set up across three Oxford deanery hospitals. Forum members were assigned classical forum roles, but were also tasked to complete quality improvement projects, organise a national quality improvement conference and undergo formal leadership and management training. Members were surveyed before and after training completion, revealing statistically significant confidence increases in leadership, management and quality improvement skills. All members revealed an eagerness to continue formal leadership and management training. The foundation forum proved a successful initiative to improve overall trainee experience and involvement, whilst providing a platform for leadership and management training to satisfy key domains within the Medical Leadership Competency Framework.

\section{FOUNDATION MENTORSHIP SCHEME PILOT PROGRAMME: IS NEAR-PEER MENTORSHIP AN EFFECTIVE WAY TO LOOK AFTER INCOMING FY1S?}

Ananth Srinivasan, Maria Tadros*, Isabel Nelson, Mark Zimmerman, Samuel Haynes, Sigourney Ducksbury, Huma Naqvi. Sandwell and West Birmingham Hospitals NHS Trust

\subsection{6/leader-2019-FMLM.16}

Background Foundation Mentorship Scheme (FMS) is a near-peer lead programme developed by Foundation Year 2 (FY2) doctors for Foundation Year 1s (FY1s) as a pastoral adjunct to the education (ES) and clinical supervisor (CS) framework.

Aims FMS aims to provide a point of contact for FY1s; to bridge the gap between FY1s and ES/CS, troubleshoot on the shop-floor, help with e-portfolio and offer pastoral support.

Methods Mentors recruited from a pool of FY2s at a District General Hospital in Birmingham UK were matched in 1:4 groups with FY1s on concurrent rotations. Two meetings were undertaken per rotation, with further meetings as necessary. Quantitative Results were obtained using online surveys and qualitative feedback provided postmeetings.

Results FMS commenced 2 months into the first rotation with 33 FY1s. Thirteen (40\%) FY1s engaged with the programme, ten $(30 \%)$ opted out, four $(12.5 \%)$ were unaware of the scheme. Five (16\%) were unable to meet their mentors. 92\% of engaging FY1s found the scheme useful, with 94\% agreeing FMS would be invaluable if commenced earlier. The themes indicated FMS was most useful during the first rotation when FY1 learning curve is steepest; highly appreciated in surgical specialties; and effective in advising on e-portfolio. Other concerns included managing difficult colleagues; under-staffing; overstaying contracted hours; and teaching. Concerns were escalated appropriately to Foundation Programme leads.

Conclusion Challenges were encountered during scheme development. A significant proportion of FY1s opted out due to late commencement of the programme. Challenges coordinating across two hospital sites contributed to drop-outs. This scheme greatly benefited the engaging FY1s. To facilitate its success, FMS should be integrated with FY1 induction with protected time reserved for mentor meetings. Mentorship is a significant responsibility and formal training should be offered to mentors, especially in handling pastoral concerns. 